\title{
Ionisation differential cross section measurements for N2 at low incident energy in coplanar and non-coplanar geometries \\ DOI:
}

10.1088/0953-4075/49/19/195202

\section{Document Version}

Accepted author manuscript

Link to publication record in Manchester Research Explorer

Citation for published version (APA):

Sakaamini, A., Amami, S., Murray, A. J., Ning, C., \& Madison, D. (2016). lonisation differential cross section measurements for $\mathrm{N}_{2}$ at low incident energy in coplanar and non-coplanar geometries. Journal of Physics $B$ : Atomic, Molecular artd Optical Physics, 49(19), [195202]. https://doi.org/10.1088/0953-4075/49/19/195202

Published in:

Journal of Physics B: Atomic, Molecular and Optical Physics

\section{Citing this paper}

Please note that where the full-text provided on Manchester Research Explorer is the Author Accepted Manuscript or Proof version this may differ from the final Published version. If citing, it is advised that you check and use the publisher's definitive version.

\section{General rights}

Copyright and moral rights for the publications made accessible in the Research Explorer are retained by the authors and/or other copyright owners and it is a condition of accessing publications that users recognise and abide by the legal requirements associated with these rights.

\section{Takedown policy}

If you believe that this document breaches copyright please refer to the University of Manchester's Takedown Procedures [http://man.ac.uk/04Y6Bo] or contact uml.scholarlycommunications@manchester.ac.uk providing relevant details, so we can investigate your claim.

\section{OPEN ACCESS}




\section{PACS No. 34.80.Dp}

\section{Introduction}

Understanding electron impact ionization of matter is important in areas ranging from plasma studies, through to detailing ionization in biology and medicine, to understanding collisions in the earth's atmosphere and in space. A complete description of the interaction requires quantum calculations to fully detail the collisions that occur. Testing of models is carried out by experiments that measure the probability of ionization as a function of all parameters that describe the interaction $[1,2]$. For single ionization by electron impact where the spins of the electrons are not detected, the interaction is fully characterised by the momentum of the incident electron $\mathbf{k}_{0}$ as well as that of the scattered and ejected electrons $\mathbf{k}_{1}$ and $\mathbf{k}_{2}$. A triple differential cross section $\operatorname{TDCS}\left(\mathbf{k}_{\mathbf{0}}, \mathbf{k}_{\mathbf{1}}, \mathbf{k}_{\mathbf{2}}\right)$ is defined, that is directly proportional to the ionization probability. This probability is determined by measuring the time-correlated signal between scattered and ejected electrons as a function of $\mathbf{k}_{\mathbf{0}}, \mathbf{k}_{\mathbf{1}}$ and $\mathbf{k}_{\mathbf{2}}$ in an (e,2e) experiment.

Following the collision, the scattered and ejected electrons may emerge over $4 \pi$ steradians, and so it is necessary to define a scattering geometry to allow the data to be compared to theory. In the experiments described here a detection plane is defined by the normal to the plane given by $\hat{\mathbf{n}}_{\mathbf{D}}=\hat{\mathbf{k}}_{1} \times \hat{\mathbf{k}}_{2}$, the incident electron making an angle $\psi$ with respect to this plane as shown in figure 
1. When $\psi=0^{\circ}$ the incident electron is in the plane so that $\hat{\mathbf{k}}_{0} \cdot \hat{\mathbf{n}}_{\mathbf{D}}=0 \cdot \hat{\mathbf{k}}_{0} \equiv \hat{\mathbf{k}}_{0}^{\psi=0^{\circ}}$ defines the quantization $\mathrm{z}$-axis in the detection plane as shown. If $\psi=90^{\circ}$ the incident electron momentum $\mathbf{k}_{0}$ is orthogonal to both outgoing electron momenta.

For experiments where the outgoing electrons emerge on opposite sides of the plane $\theta_{1}=\theta_{2}=90^{\circ}$.

Under these conditions the detection plane is no longer well defined (since $\hat{\mathbf{k}}_{1} \times \hat{\mathbf{k}}_{\mathbf{2}}=0$ ) and so the TDCS is independent of the angle $\psi$. A common point hence exists for all angles $\psi$ when $\theta_{1}=\theta_{2}=90^{\circ}$, so that at any given incident energy the data can be inter-normalised at this point.

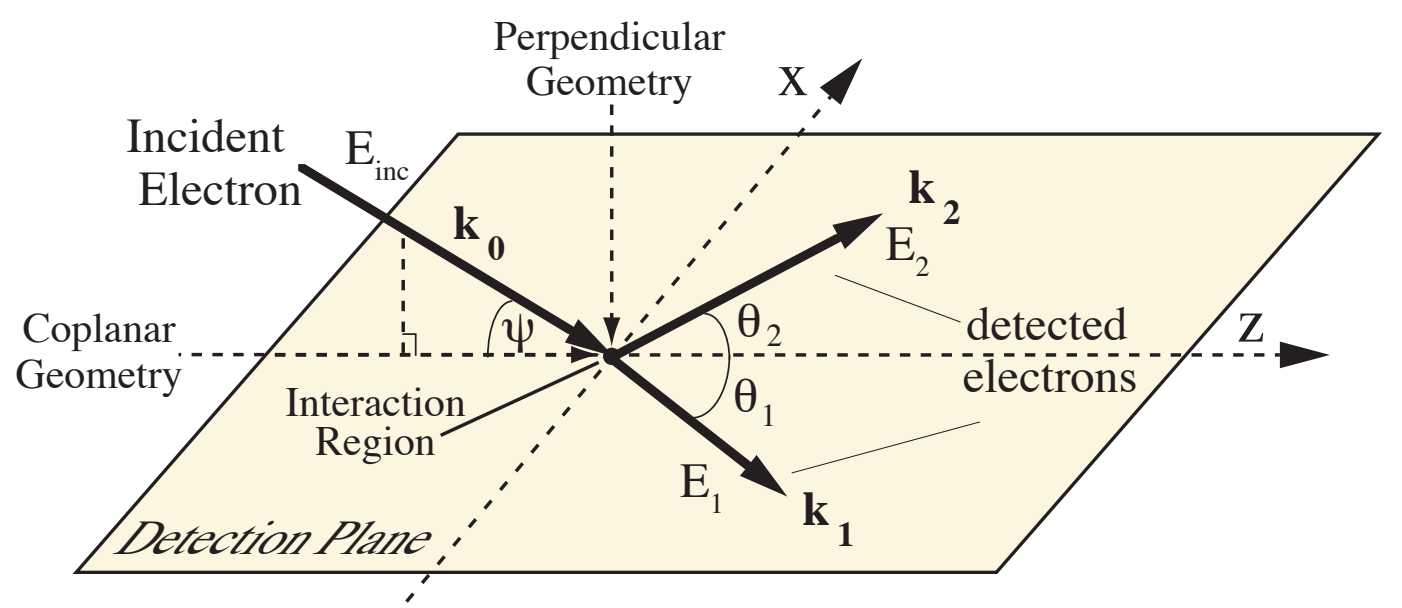

Fig 1. The experimental geometry adopted in this work. For details see text.

The (e,2e) spectrometer in Manchester allows $\psi$ to vary from $0^{\circ}$ to $90^{\circ}$. The outgoing electrons can be detected from $\theta_{1,2}=35^{\circ}$ to $125^{\circ}$ when $\psi=0^{\circ}$ to $70^{\circ}$, this range extending to $\theta_{1,2}=35^{\circ}$ to $140^{\circ}$ when $\psi>70^{\circ}$. These constraints are due to the physical size of the electron gun and electron detectors.

$\mathrm{N}_{2}$ is the lightest diatomic molecule apart from $\mathrm{H}_{2}$ that can easily be studied, since a molecular beam of $\mathrm{N}_{2}$ can be delivered to the interaction region from a gas needle. $\mathrm{H}_{2}$ has been extensively investigated both experimentally and theoretically [3-7], leading to considerable progress in understanding the dynamics of the ionizing collision with a molecular target. Models of the interactions include both time-independent studies using distorted wave calculations [3], as well as time-dependent models that use close-coupling techniques [4]. These models have been tested by experiment in both high and low energy regimes, under a wide variety of kinematic conditions [5-7]. Modelling the ionization of molecules by electron impact is considerably more complex than for atomic targets, since they can have internal energy in rotational and vibrational motion, and their electronic structure is inherently non-spherical due to their distributed nuclei. Almost all (e,2e) experiments carried out so far do not determine the nuclear orientation during the collision, and so 
the calculations must average over all possible orientations of the target. The energy resolution in most experiments is also insufficient to resolve the rotational and vibrational motion of the target, and so it is necessary to further average over the different states that may contribute. It is possible to determine individual vibrational contributions from selected targets using energy-selected electron guns, however very few studies have been carried out so far due to the low coincidence yield in these experiments [8].

Several (e,2e) experiments and theoretical calculations have already been carried out from an $\mathrm{N}_{2}$ target, however these have mostly been in an asymmetric coplanar geometry at medium to high incident energies [9-16]. By contrast, the work presented here is at low energies in a symmetric configuration. In this regime the cross section is highly sensitive to different collision process including exchange, distortions to the wave-fronts describing the incident, scattered and ejected electrons, target polarization and post collisional interactions as the electrons leave the interaction region. Calculation of the cross sections in this energy region is hence very challenging, as approximations that are often adopted at higher energies cannot be used.

This paper is divided into five sections. Following this introduction the experimental procedures used to measure the data are briefly described. A description of the theoretical models used to calculate the relevant cross sections is then presented. Section 4 compares the data to results from three different distorted wave models, and differences between these are discussed. Section 5 then summarises these studies so far, and considers the next steps that are required in these investigations.

\section{Experimental Procedures.}

$\mathrm{N}_{2}$ is a stable diatomic molecule that has 10 valence electrons and 4 core electrons. The valence electrons combine to produce the strong triple bond in the $\mathrm{N}_{2}$ molecule, the electrons pairing to form the $3 \sigma_{g}^{2}, 1 \pi_{u}^{4}$ and $2 \sigma_{g}^{2}$ bonding orbitals and the $2 \sigma_{u}^{2}$ anti-bonding orbital. The $2 \sigma_{g}^{2}$ orbital is the deepest valence state, and has a binding energy more than $20 \mathrm{eV}$ higher than that of the $2 \sigma_{u}^{2}$ orbital. The ground state electronic configuration of $\mathrm{N}_{2}$ is hence $\left(2 \sigma_{g}^{2} 2 \sigma_{u}^{2} 1 \pi_{u}^{4} 3 \sigma_{g}^{2}\right)^{1} \Sigma_{g}^{+}$. Ionization can occur from each orbital, leading to $N_{2}^{+}$ions in different final states. In the work presented here measurements were taken from the three outer orbitals. This produces the $X^{2} \Sigma_{g}^{+} N_{2}^{+}$state for ionization from the $3 \sigma_{g}$ orbital, the $A^{2} \Pi_{u}$ state when electrons are ejected from the $1 \pi_{u}$ orbital, and the $B^{2} \Sigma_{u}^{+}$state for ejection of electrons from the $2 \sigma_{u}$ orbital.

An example of the ionization binding energy spectrum produced from different orbitals is shown in figure 2, taken with the $(\mathrm{e}, 2 \mathrm{e})$ spectrometer in Manchester. The incident electron gun was 
unselected in energy, and the electron momentum analysers were set to optimise the signal energy resolution while ensuring the best yield for coincidence counting. In this example, the electron analysers each detected electrons with an energy $\sim 4.6 \mathrm{eV}$, and coincidence counts were measured at angles $\theta_{1}=\theta_{2}=45^{\circ}$. The figure shows the results from three different incident electron angles with (a) $\psi=0^{\circ}$, (b) $\psi=45^{\circ}$ and (c) $\psi=90^{\circ}$. The data were obtained by measuring the coincidence yield over a range of incident energies from $23 \mathrm{eV}$ to $29 \mathrm{eV}$, in steps of $0.125 \mathrm{eV}$. The data were accumulated for 5000 seconds at each energy, and the results were then normalised to unity at the peak of the $3 \sigma_{g}$ state in a coplanar geometry.

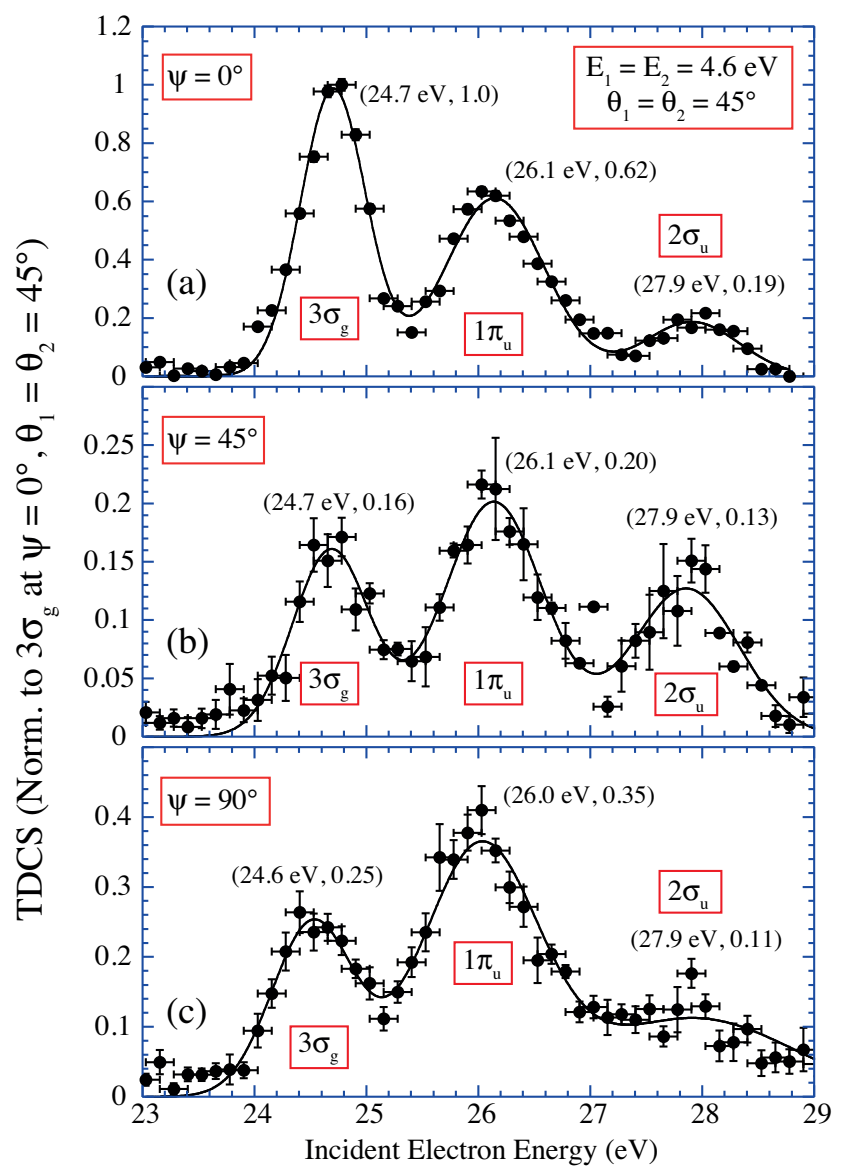

Fig 2. Binding energy spectra for outgoing electron energies of $4.6 \mathrm{eV} \pm 0.5 \mathrm{eV}$ taken with the electrons detected at a forward angle of $45^{\circ}$ to the z-axis. The results are shown for (a) the coplanar geometry, (b) for the incident electron at $\psi=45^{\circ}$ to the detection plane, and (c) for the perpendicular geometry $\left(\psi=90^{\circ}\right)$. Gaussians are fitted to the data for each state, so that the relative contributions and their peak energies could be determined. The peak of the $3 \sigma_{g}$ state in a coplanar geometry was set to unity, and all data were then inter-normalised to this peak as discussed in the text.

Figure 2 demonstrates that the experimental apparatus can resolve the contribution from each of the orbitals of the molecule, and shows that the cross-section depends on the individual states that are ionized. The spectral scans at each angle $\psi$ were taken under the same operating conditions, and so were used to inter-normalise the results from each of the individual orbitals. The data at different angles $\psi$ were then inter-normalised through the common point at $\theta_{1}=\theta_{2}=90^{\circ}$. Binding energy 
spectra similar to figure 2 were taken for each of the data sets that were measured, so that all data at any given energy could be normalised to a common point.

Three sets of coincidence data were taken for outgoing electron energies of $(4.6 \mathrm{eV}, 4.6 \mathrm{eV})$, $(9.7 \mathrm{eV}, 9.7 \mathrm{eV})$ and $(14.5 \mathrm{eV}, 4.6 \mathrm{eV})$, with incident beam angles of $\psi=0^{\circ}, 45^{\circ}$ and $90^{\circ} .27$ individual angular data sets for the TDCS were hence obtained during this study. The data were accumulated using up to 10 sweeps of the detection plane, with coincidence measurements being taken typically for 5000 seconds at each scattering angle. In all of the data sets a symmetric geometry was chosen, so that $\theta_{1}=\theta_{2}=\theta$. The measurements at each angle $\theta$ were then averaged, and their respective uncertainties calculated from the standard error on the mean. In each data set the cross sections were inter-normalised to the peak of the $3 \sigma_{g}$ in a coplanar geometry as discussed above. All experimental TDCS measurements at any given energy were hence placed on a common scale.

The energy of the electrons emitted from the electron gun were calibrated against the $19.337 \mathrm{eV}$ elastic resonance in helium [17], whereas the energies of the scattered and ejected electrons detected by the electron energy analysers were determined from inelastic scattering resonances in this target. Helium was chosen for this calibration as its resonances are very well known [18], and since it has no ro-vibrational structure. These energy calibrations were carried out both before and after each set of measurements were made, so as to allow for any variation in the spectrometer operating conditions over time.

The typical operating pressure in the vacuum chamber during data accumulation was $\sim 2 \times 10^{-5}$ torr, with a base pressure of $1 \times 10^{-7}$ torr. The incident electron beam current was set at $200 \mathrm{nA}$ so that the coincidence signal could be easily resolved from the background. The timing window of the Time to Amplitude converter was set to $500 \mathrm{~ns}$, and a delay time of $\sim 300 \mathrm{~ns}$ was added to the stop signal so that the coincidence peak was positioned close to the centre of the timing spectrum. The spectrometer operated under computer control, the analyser tuning conditions being optimised each time the analysers were moved to a new angle. In this way changes in the operating conditions of the spectrometer as the experiments proceeded could be minimised. Full details of the computer control and optimisation systems used in these experiments can be found in [19]. 


\section{Theory.}

We have used three different theoretical models to calculate the TDCS for the $\mathrm{N}_{2}$ molecule - the molecular three-body distorted wave (M3DW) approximation, the distorted wave Born approximation (DWBA), and the DWBA using the Ward-Macek (WM) approximation for the final state electron-electron interaction, which is normally called the post-collision interaction (PCI). All these approaches have been described in detail previously in several references [2-3] and [7, 11, 20, 21]. Here we will only give a brief summary of the models to show their differences and similarities. Because the DWBA is a special case of the more general M3DW approximation, we will start our discussion with the M3DW.

\subsection{The M3DW Approximation}

The M3DW direct scattering $T$-matrix can be written as,

$$
T_{d i r}^{M 3 D W}=\left\langle\Psi_{f}|W| \Psi_{i}\right\rangle
$$

Here $\Psi_{f}$ and $\Psi_{i}$ are the final- and initial-state wave functions for the system respectively, and $W$ is the perturbation. The initial-state wavefunction $\Psi_{i}$ is approximated as a product of a distorted wave function $\chi_{0}^{+}\left(\mathbf{r}_{1}\right)$ for the incoming electron (the projectile) times the initial Dyson bound state wavefunction $\phi_{D y}\left(\mathbf{r}_{2}\right)$ for the target $\mathrm{N}_{2}$ molecule which is averaged over all molecular orientations.

$$
\Psi_{i}=\chi_{0}^{+}\left(\mathbf{r}_{1}\right) \phi_{D y}\left(\mathbf{r}_{2}\right)
$$

The final-state wavefunction $\Psi_{f}$ for the two outgoing electrons, called the scattered and ejected electrons for convenience, is approximated as a product of two final-state continuum electron distorted waves $\left(\chi_{1}^{-}\left(\mathbf{r}_{1}\right)\right.$ and $\left.\chi_{2}^{-}\left(\mathbf{r}_{2}\right)\right)$, and the Coulomb interaction between the outgoing electrons $\left(C_{12}\left(\mathbf{r}_{12}, \mathbf{k}_{12}\right)\right)$, so that

$$
\Psi_{f}=\chi_{1}^{-}\left(\mathbf{r}_{1}\right) \chi_{2}^{-}\left(\mathbf{r}_{2}\right) C_{12}\left(\mathbf{r}_{12}, \mathbf{k}_{12}\right)
$$

where

$$
C_{12}\left(\mathbf{r}_{12}, \mathbf{k}_{12}\right)=e^{-\pi \gamma / 2} \Gamma(1-i \gamma)_{1} F_{1}\left(i \gamma, 1,-i\left[k_{12} r_{12}+\mathbf{k}_{12} \bullet \mathbf{r}_{12}\right]\right)
$$

Here $\Gamma(1-i \gamma)$ is a gamma factor, ${ }_{1} F_{1}$ is a confluent hyper-geometric function, $r_{12}$ is the relative distance between the two electrons, $v_{12}$ is the relative velocity between the two continuum electrons, and $k_{12}=\mu v_{12}$ where $k_{12}$ is the relative momentum. $\mu=1 / 2$ is the reduced mass for the two 
electrons in atomic units, and $\gamma=1 / v_{12}$ is the Sommerfeld parameter. When the PCI term is included directly in the final state wavefunction, PCI is included to all orders of perturbation theory.

Finally the perturbation $W$ is given by,

$$
W=V-U_{i}
$$

where $V$ is the exact initial state interaction between the neutral molecule and the projectile (in this case the incident electron), and $U_{i}$ is an initial state spherically symmetric approximation for $V$.

With these approximations, the direct M3DW T-matrix becomes

$$
T_{d i r}^{M 3 D W}=\left\langle\chi_{1}^{-}\left(\mathbf{r}_{1}\right) \chi_{2}^{-}\left(\mathbf{r}_{2}\right) C_{12}\left(\mathbf{r}_{12}, \mathbf{k}_{12}\right)|W| \chi_{0}^{+}\left(\mathbf{r}_{1}\right) \phi_{D y}\left(\mathbf{r}_{2}\right)\right\rangle
$$

The M3DW approximation has been shown to give very good agreement with experiment for ionization of $\mathrm{H}_{2}$ [7, 22] for energies down to threshold and for $\mathrm{N}_{2}[14,20,23,24]$ for higher incident energy electrons.

\subsection{The DWBA Approximation}

In the T-matrix of the standard DWBA approximation, the interaction between the two continuum electrons in the final state $C_{12}$ is omitted in the approximation for the final-state wavefunction. Thus the DWBA $T$-matrix is

$T_{d i r}^{D W B A}=\left\langle\chi_{1}^{-}\left(\mathbf{r}_{1}\right) \chi_{2}^{-}\left(\mathbf{r}_{2}\right)|W| \chi_{0}^{+}\left(\mathbf{r}_{1}\right) \phi_{D y}\left(\mathbf{r}_{2}\right)\right\rangle$

In the DWBA, PCI is included only to first order.

\subsection{The WM Approximation}

In this approximation, the Ward-Macek (WM) approximation for PCI has been used [25] for the Coulomb interaction of Eq. (4). In the WM approximation, the term $\left[k_{12} r_{12}+\mathbf{k}_{12} \bullet \mathbf{r}_{12}\right]$ in the hypergeometric function is replaced by $2 k_{12} r_{12}^{\text {ave }}$, where $r_{12}^{\text {ave }}$ is the average value of the electron-electron separation.

$$
C_{12}^{W M}\left(r_{\text {ave }}, k_{12}\right)=e^{\frac{-\pi \gamma}{2}} \Gamma(1-i \gamma)_{1} F_{1}\left(i \gamma, 1,-2 i k_{12} r_{\text {ave }}\right)
$$

Since this factor does not depend on the coordinates being integrated, it can be factored from the integral in the T-matrix and the direct WM T-matrix becomes

$T_{d i r}^{W M}=C_{12}^{W M}\left(r_{a v e}, k_{12}\right)\left\langle\chi_{1}^{-}\left(\mathbf{r}_{1}\right) \chi_{2}^{-}\left(\mathbf{r}_{2}\right)|W| \chi_{0}^{+}\left(\mathbf{r}_{1}\right) \phi_{D y}\left(\mathbf{r}_{2}\right)\right\rangle$ 
$T_{d i r}^{W M}=C_{12}^{W M}\left(r_{a v e}, k_{12}\right) T_{d i r}^{D W B A}$

Finally in all three models, the triple differential cross section (TDCS) in atomic units can be written as,

$$
T D C S=\frac{d^{5} \sigma}{d \Omega_{1} d \Omega_{2} d E_{2}}=\frac{1}{(2 \pi)^{5}} \frac{k_{1} k_{2}}{k_{0}}\left(\left|T_{d i r}\right|^{2}+\left|T_{e x c}\right|^{2}+\left|T_{d i r}-T_{e x c}\right|^{2}\right),
$$

Here $k_{0}, k_{1}$, and $k_{2}$ are the magnitudes of the momenta of the initial, the scattered, and the ejected electrons, respectively and $T_{e x c}$ is the exchange T-matrix which is calculated similar to $T_{d i r}$ except that the two final state electrons are interchanged in the final state wavefunction $\Psi_{f}$.

\section{Comparison of theory to experiment.}

Figures 3-5 show the complete set of data compared to the three different models described in section 3. Figures 3 and 4 show results when $E_{1}=E_{2}$, whereas figure 5 shows data when $E_{1} \neq E_{2}$. Figure 3 is for outgoing electron energies of $4.6 \mathrm{eV} \pm 0.5 \mathrm{eV}$, figure 4 shows results when $E_{1}=E_{2}=9.7 \mathrm{eV}$, and figure 5 shows results for $E_{1}=14.5 \mathrm{eV}, E_{2}=4.6 \mathrm{eV}$. In all cases $\theta_{1}=\theta_{2}=\theta$.

Since the experiments did not measure an absolute cross section, the maximum in the data for the $3 \sigma_{g}$ state has been normalised to the peak of the M3DW theory in a coplanar geometry at each energy. All other experimental data in each figure were then inter-normalised to this peak, as described above. The common point at $\theta_{1}=\theta_{2}=90^{\circ}$ is highlighted with a red circle in each figure. The calculated theoretical cross sections are all on an absolute scale, and so they could be directly compared to each other.

Nine sets of data are shown in each figure. The first column shows the results from ionizing the $3 \sigma_{g}$ state for (a) $\psi=0^{\circ}$, (b) $\psi=45^{\circ}$ and (c) $\psi=90^{\circ}$. The middle column shows results from the $1 \pi_{u}$ state, whereas the final column shows results from the $2 \sigma_{u}$ state. The M3DW calculation is shown as a solid black curve, the DWBA calculation is shown as a red coarse-dashed curve, and the calculation that includes the Ward-Macek interaction term for PCI is shown as a blue finely-dashed curve. In all cases when the outgoing electrons have equal energy (as in figures 3 and 4), PCI between the electrons force the TDCS to be zero at $\theta=0^{\circ}$ and $180^{\circ}$. This can be seen most clearly for both the M3DW and WM models. 


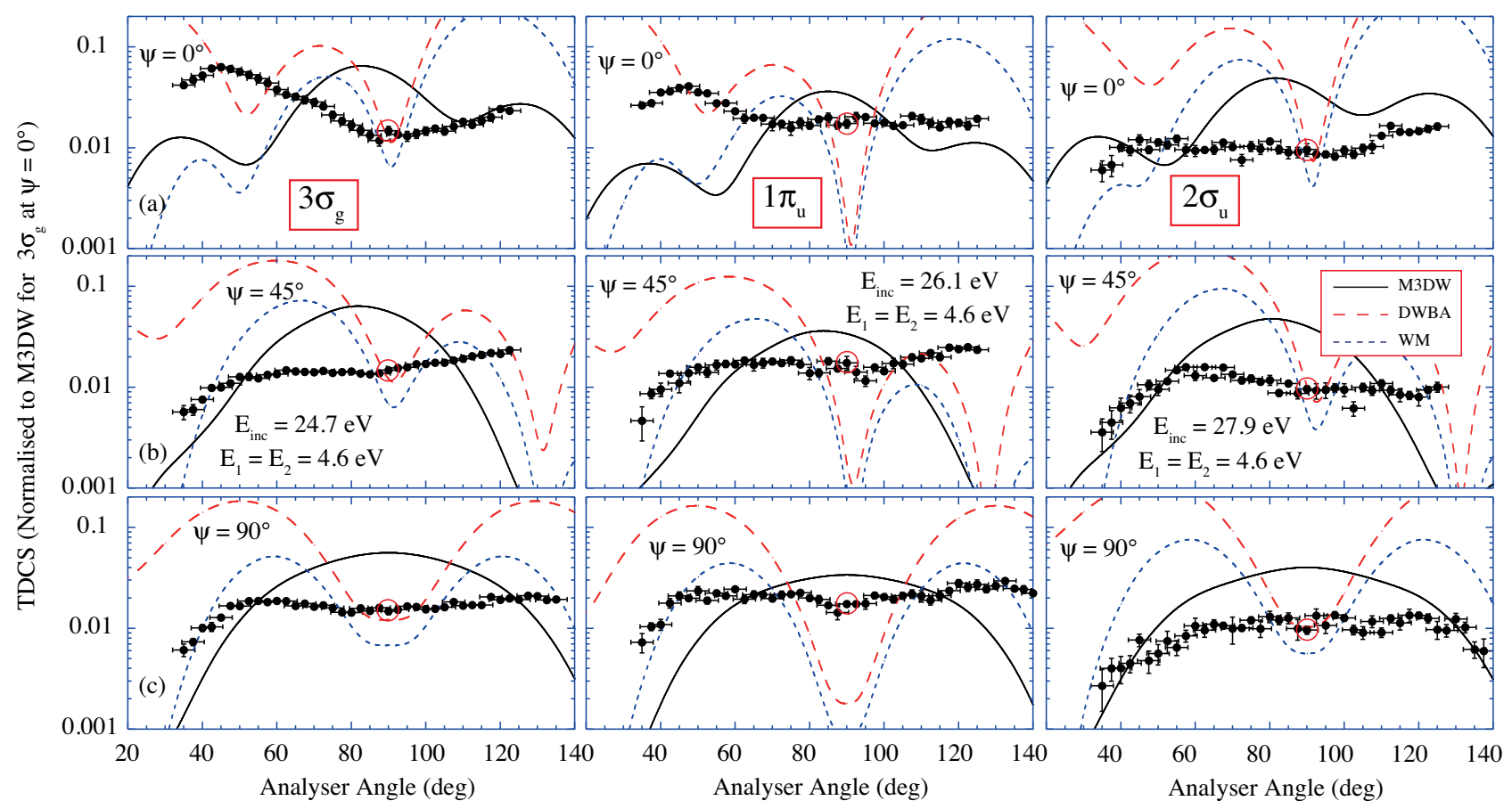

Fig 3. Normalised TDCS data for outgoing electron energies of $4.6 \mathrm{eV} \pm 0.5 \mathrm{eV}$ in (a) a coplanar geometry, (b) for the incident electron at $45^{\circ}$ to the detection plane, and (c) for the perpendicular geometry. The peak of the experimental data for the $3 \sigma_{g}$ state are set equal to that of the M3DW calculations. The coplanar data for the $1 \pi_{u}$ and $2 \sigma_{u}$ states are then set relative to the $3 \sigma_{g}$ state using the binding energy spectra in figure 2 . The common normalisation point when $\theta_{1}=\theta_{2}=90^{\circ}$ is then used to inter-normalise all data sets which are plotted on a logarithmic scale.

Figure 3 shows the results for outgoing electron energies of $4.6 \mathrm{eV} \pm 0.5 \mathrm{eV}$ plotted on a logarithmic scale. The coplanar data for the $3 \sigma_{g}$ state indicate that the TDCS is dominated by forward scattering in this geometry, with the peak in the cross section being found at $\theta \sim 45^{\circ}$. A minimum occurs at $\theta \sim 90^{\circ}$, and the cross section then increases again at higher scattering angles.

When the incident electron beam is raised out of the plane, the measured TDCS is no longer dominated by forward scattering. The $3 \sigma_{g}$ data for $\psi=45^{\circ}$ indicates that the electrons are preferentially back scattered at this energy, with the cross section being relatively uniform as the scattering angle changes.

The TDCS in the perpendicular plane must be symmetric around $\theta=90^{\circ}$, and this is borne out in the data. As for the results at $\psi=45^{\circ}$, the measured TDCS shows little structural change under these conditions, and is largely uniform in magnitude over a wide range of scattering angles.

The experimental data for the $1 \pi_{u}$ state follows a similar trend to that of the $3 \sigma_{g}$ state, with a maximum in the forward direction for a coplanar geometry, and a slight backscattering dominance when $\psi=45^{\circ}$. The coplanar forward peak and backscatter peaks are however not as pronounced as for the $3 \sigma_{g}$ state. By contrast, the data from the $2 \sigma_{u}$ state changes little as the incident electron 
beam direction is changed. In all cases the TDCS measurements for this state are relatively uniform as the scattering angles changed in the experiment, indicating that the scattering dynamics from the $2 \sigma_{u}$ state has no particular preference for either forward or backward scattering.

The theoretical calculations at this energy show a very different trend to the experimental data. The DWBA calculation does not include PCI to all orders, and the large difference between this calculation and the data clearly shows the importance of its inclusion. In all cases the DWBA theory over-estimates the cross section, and predicts features that are not seen in the data. Inclusion of the Ward-Macek (WM) interaction significantly improves the results compared to experiment, however once again this calculation predicts structures that are not observed. The WM theory does however produce peaks that are in the same position as the data in non-coplanar geometries.

The M3DW calculation includes the effects of PCI exactly, and so it would seem that it should provide the most accurate estimate of post-collisional interactions. Somewhat surprisingly this calculation does not improve the results from the WM theory, but rather predicts structures that are in disagreement with the data in all cases. This theory predicts that the TDCS should be dominated by a peak near $\theta=90^{\circ}$, as was found for helium at similar energies [26]. It would appear that at these energies the M3DW theory is including PCI too strongly, compared to other scattering processes that lead to ionization. In earlier works for low energy ionization of $\mathrm{H}_{2}$, we also found that the M3DW overestimated the effects of PCI and that the WM model agreed better with experiment [7]. It is also interesting to note that the M3DW calculation predicts a maximum when $\theta=90^{\circ}$ in the perpendicular plane, in contrast to the WM and DWBA theories that predict a minimum.

Figure 4 details the results for equal outgoing electron energies of $9.7 \mathrm{eV} \pm 0.5 \mathrm{eV}$, and show a much improved comparison between theory and experiment, particularly for the M3DW calculation. Once again for the $3 \sigma_{g}$ and $1 \pi_{u}$ states, forward scattering dominates in a coplanar geometry. The data for $\psi=45^{\circ}$ also has more structure than at the lower energy for these states. In the perpendicular plane the data are again broadly featureless as a function of scattering angle, although the data from the $1 \pi_{u}$ state appears to have two broad peaks with a minimum at $\theta=90^{\circ}$. The data for the $2 \sigma_{u}$ state again shows very little change with either scattering angle or incident angle at this energy. 


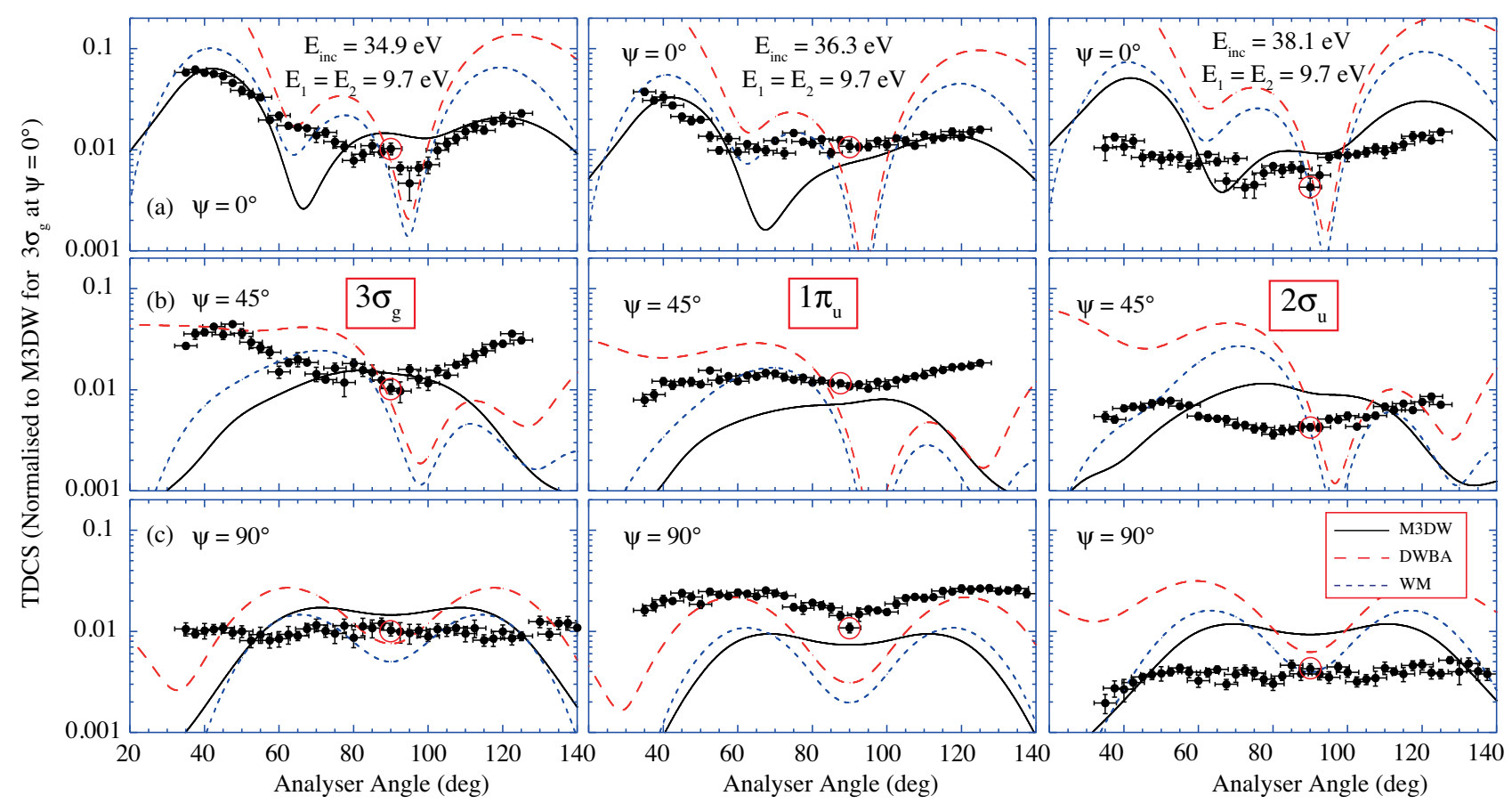

Fig 4. Normalised TDCS data for outgoing electron energies of $9.7 \mathrm{eV} \pm 0.5 \mathrm{eV}$ taken in (a) a coplanar geometry, (b) for the incident electron at $45^{\circ}$ to the detection plane, and (c) for a perpendicular geometry. The peak of the experimental data for the $3 \sigma_{g}$ state are set to that of the M3DW calculations at this energy. The data are then internormalised as described in figure 3.

At this energy the DWBA theory again fails to predict the measured TDCS, however inclusion of the Ward-Macek approximation for PCI now greatly improves this comparison. The M3DW calculation is the most accurate in the coplanar geometry, with the calculation closely emulating the data for both the $3 \sigma_{g}$ and $1 \pi_{u}$ states. None of the calculations predict the results for the $2 \sigma_{u}$ state in this geometry. As the incident electron beam is raised out of the scattering plane the calculations more closely emulate the magnitude of the data compared to the lower energy results in figure 3 . The WM calculation more closely emulates the data for both the $3 \sigma_{g}$ and $1 \pi_{u}$ states under these conditions, although none of the calculations predict the data accurately. The calculations for the $2 \sigma_{u}$ state do not agree with the data for any of the incident electron angles.

The final set of results for all three states shown in figure 5 relax the 'doubly symmetric' conditions of the experiment and choose different energies of the outgoing electrons, with $E_{1}=14.5 \mathrm{eV}$ and $E_{2}=4.6 \mathrm{eV}$. The incident electron energies are hence similar to that chosen for the measurements in figure 4, with the incident energies set by fitting to the data in the binding energy spectrum under these conditions. Once again the data for the $3 \sigma_{g}$ and $1 \pi_{u}$ states in a coplanar geometry are dominated by forward scattering, however the peaks are less pronounced than when the outgoing electron energies are equal. The results when $\psi=45^{\circ}$ for these states lie somewhere between those found in figures 3 and 4, whereas the data in the perpendicular plane is once again broadly 
featureless as the scattering angle changes. The data for the innermost $2 \sigma_{u}$ state again shows little variation as both $\theta$ and $\psi$ are varied.

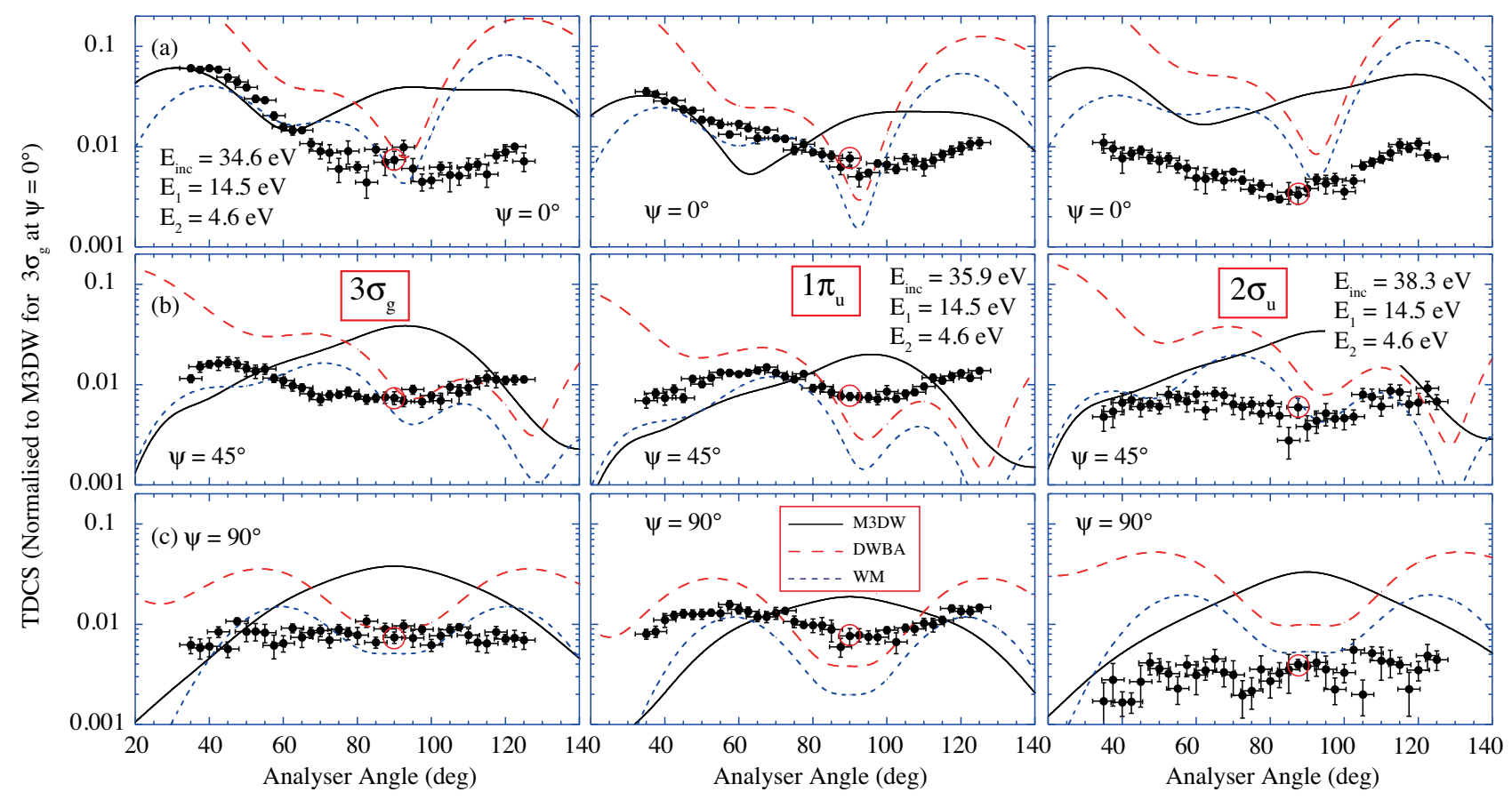

Fig 5. Normalised TDCS data for outgoing electron energies of $4.6 \mathrm{eV} \pm 0.5 \mathrm{eV}$ and $14.5 \mathrm{eV} \pm 0.5 \mathrm{eV}$ taken in (a) a coplanar geometry, (b) for the incident electron at $45^{\circ}$ to the detection plane, and (c) for the perpendicular geometry. The peak of the experimental data for the $3 \sigma_{g}$ state are set to that of the M3DW calculations at this energy. The data are then inter-normalised as described in figures $3 \& 4$.

The DWBA calculation once more fails to predict the data, whereas the WM calculation now agrees most closely with the results from experiment for the $3 \sigma_{g}$ and $1 \pi_{u}$ states. The M3DW calculation again appears to overestimate the effects of PCI, producing a dominant peak at $\theta=90^{\circ}$ in the perpendicular plane that is not seen in the data. Both WM and M3DW calculations predict a higher coplanar cross section in the backward direction than is found in the data. The WM calculation more closely emulates the data under non-coplanar conditions. In all cases the calculations again do not predict the results from the $2 \sigma_{u}$ state.

The final set of results shown in figure 6 are for ionization from the $3 \sigma_{g}$ state at an incident energy $\sim 40 \mathrm{eV}$ above the ionization potential, to ascertain how each model compares to experiment at this higher energy. No data were taken for the $1 \pi_{u}$ or $2 \sigma_{u}$ states under these conditions.

There is an overall improvement in the coplanar geometry compared to the results at lower energies, particularly in the forward direction where both M3DW and WM models closely emulate the position of the peak in the TDCS. This comparison is less satisfactory at higher scattering angles in this plane. By contrast, the DWBA model agrees most closely with the data for $\psi=45^{\circ}$, with both M3DW and WM models underestimating the cross section under these conditions. The agreement for all models is better in the perpendicular plane, although all underestimate the cross section when 
compared to the data. Overall, it appears that the models are becoming progressively more accurate as the incident energy increases.

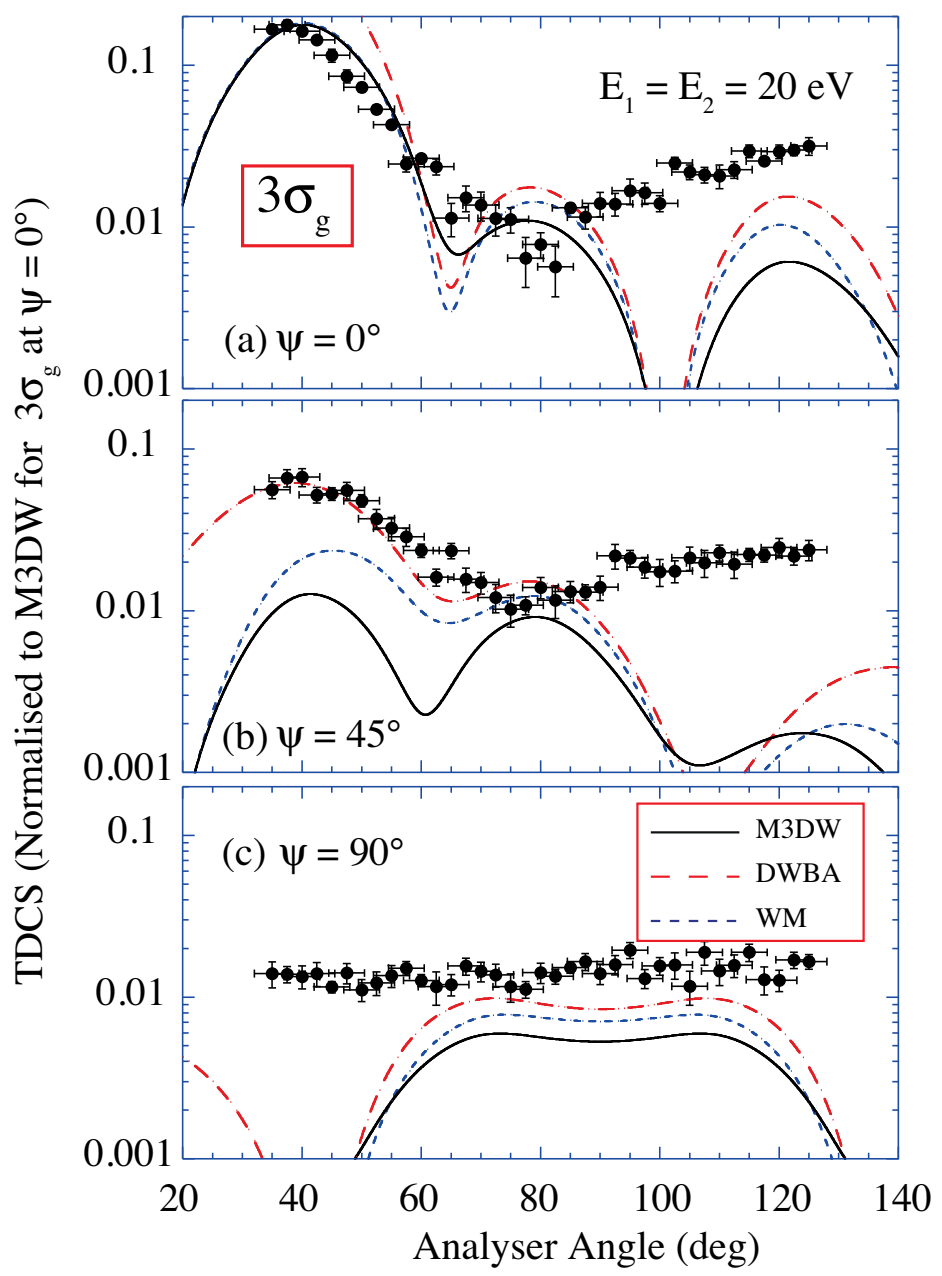

Fig 6. Normalised TDCS data for equal outgoing electron energies of $20 \mathrm{eV} \pm 0.5 \mathrm{eV}$ taken in (a) a coplanar geometry, (b) for the incident electron at $45^{\circ}$ to the detection plane, and (c) for the perpendicular geometry. The peak of the experimental data for the $3 \sigma_{g}$ state are set to that of the M3DW calculations at this energy. The data are then internormalised to the common point.

\section{Discussion and conclusions.}

The results from these experiments on $\mathrm{N}_{2}$ show that the ionization cross-section is very sensitive to both the state from which the ionization occurs, and the collisional energy of the interaction. The data have been presented over a range of angles from a coplanar geometry through to the perpendicular plane, allowing the data at any given energy to be normalised to a single point. Binding energy spectra were also taken that allowed data from the outermost $3 \sigma_{g}, 1 \pi_{u}$ and $2 \sigma_{u}$ states to be inter-normalised. These data have been presented for outgoing electron energies $E_{1}=E_{2}=4.6 \mathrm{eV}, E_{1}=E_{2}=9.7 \mathrm{eV}$ and $E_{1}=14.5 \mathrm{eV}, E_{2}=4.6 \mathrm{eV}$. Calculation based upon distorted wave methods have also been shown, with three different theories showing the sensitivity of PCI to the scattering process. The importance of post collisional interactions in the model have 
been demonstrated, with both the Ward-Macek approximation and a full three-body calculation having been used. It is found that in some cases the full three-body calculation overestimates the effects of PCI, and that the WM approximation proves more accurate. A similar observation was found in earlier low energy ionization of $\mathrm{H}_{2}$. Theory more closely approaches the data as the energy is increased, and so it will be interesting to see if further increases in the energy will improve these comparisons. Additional experiments to test this hypothesis are currently underway.

\section{Acknowledgements.}

AS would like to thank the University of Manchester for financial support through their overseas student award. SA and DM would like to acknowledge the support of the US National Science Foundation under Grant. No. PHY-1505819 and CN would like to acknowledge the support of the National Natural Science Foundation of China under Grant No. 11174175. 


\section{References.}

[1] E Weigold and I E McCarthy, Electron Momentum Spectroscopy Kluwer, Dordrecht, (1999)

[2] D. H. Madison and O. Al-Hagan, J At. Mol. Opt. Phys. 2010, 367180 (2010).

[3] J Colgan, O Al-Hagan, D H Madison, C Kaiser, A J Murray and M S Pindzola Phys. Rev. A 79 052704 (2009)

[4] J Colgan, M S Pindzola, F Robicheaux, C Kaiser, A J Murray and D H Madison Phys. Rev. Lett. $101233201(2008)$

[5] E M Staicu-Casagrande, A Naja, F Mezdari, A Lahmam-Bennani, P Bolognesi, B Joulakian, O Chuluunbaatar, O Al-Hagan, D H Madison D V Fursa and I Bray J Phys B 41025204 (2008)

[6] X Ren, A Senftleben, T Pflüger, A Dorn, J Colgan, M S Pindzola, O Al-Hagan, D H Madison, I Bray, D V Fursa and J Ullrich Phys. Rev. A 82032712 (2010)

[7] O Al-Hagan, A J Murray, C Kaiser, J Colgan and D H Madison, Phys. Rev. A 81030701 (2010)

[8] J P Doering and J Yang Phys Rev A 602176 (1999)

[9] I Toth and L Nagy J Phys B 44195205 (2011)

[10] A Naja, E M Staicu-Casagrande, A Lahmam-Bennani, M Nekkab, F Mezdari, B Joulakian, O Chuluunbaatar and D H Madison J Phys B 403775 (2007)

[11] J Gao, D H Madison and J L Peacher Phys Rev A 72032721 (2005)

[12] A Lahmam-Bennani, E M Staicu-Casagrande and A Naja J Phys B 42235205 (2009)

[13] L Avaldi, R Camilloni, E Fainelli and G Stefani J Phys B 253551 (1992)

[14] A J Murray, M J Hussey J Gao and D H Madison J Phys B 393945 (2006)

[15] A J Murray, M J Hussey, C Kaiser, J Gao and D H Madison J. Elect. Spect. 16111 (2007)

[16] A J Murray J Phys B 48245203 (2015)

[17] J N H Brunt, G C King and F H Read J Phys B 101289 (1977)

[18] A Kramida, Y Ralchenko, J Reader and NIST ASD Team (2015). NIST Atomic Spectra Database. http://physics.nist.gov/asd

[19] A J Murray, B C H Turton and F H Read Rev. Sci. Inst. 633346 (1992)

[20] J. F. Gao, D. H. Madison, and J. L. Peacher, J. Chem. Phys. 123, 204314 (2005).

[21] K L Nixon, A J Murray, H Chaluvadi, S Amami, D H Madison, and C Ning J. Chem. Phys.

136, 094302 (2012) 
[22] O Al-Hagan, C Kaiser, D Madison and A Murray, Nature Physics 5, 59 (2009)

[23] J Gao, D H Madison and J L Peacher, Phys. Rev. A Rapid Communications 72, 020701, (2005).

[24] L R Hargreaves, C Colyer, M A Stevenson, B Lohmann, O Al-Hagan, D H Madison, and C G Ning, Phys. Rev. A 80, 062704 (2009)

[25] S J Ward and J H Macek Phys. Rev. A 491049 (1994)

[26] A J Murray, F H Read and N J Bowring J Phys B 30387 (1997) 